\title{
On Free Vibrations of Elastodynamic Problem in Rotating Non-Homogeneous Orthotropic Hollow Sphere
}

\author{
S. R. Mahmoud, ${ }^{1,2}$ M. Marin, ${ }^{3}$ S. I. Ali, ${ }^{4,5}$ and K. S. Al-Basyouni ${ }^{1}$ \\ ${ }^{1}$ Department of Mathematics, Science Faculty, King Abdulaziz University, P.O. Box 80203, Jeddah 21589, Saudi Arabia \\ ${ }^{2}$ Mathematics Department, Science Faculty, Sohag University, Sohag 82524, Egypt \\ ${ }^{3}$ Mathematics Department, Faculty of Mathematics, Brasov University, Brasov, Romania \\ ${ }^{4}$ Department of Mathematics, Science Faculty, Hail University, Saudi Arabia \\ ${ }^{5}$ Department of Mathematics, Science Faculty, Al-Azhar University, Nassr City, Egypt
}

Correspondence should be addressed to K. S. Al-Basyouni; kalbasyouni@kau.edu.sa

Received 30 April 2013; Accepted 23 June 2013

Academic Editor: Abdelouahed Tounsi

Copyright (c) 2013 S. R. Mahmoud et al. This is an open access article distributed under the Creative Commons Attribution License, which permits unrestricted use, distribution, and reproduction in any medium, provided the original work is properly cited.

The effect of non-homogenity and rotation on the free vibrations for elastodynamic problem of orthotropic hollow sphere is discussed. The free vibrations are studied on the basis of the linear elasticity. The determination is concerned with the eigenvalues of the natural frequency for mixed boundary conditions. The numerical results of the frequency equations are discussed in the presence and absence of non-homogenity and rotation. The computer simulated results indicate that the influence of non-homogenity and rotation in orthotropic material is pronounced.

\section{Introduction}

Hollow spheres are frequently encountered in engineering industries and the corresponding free vibration problem has become one of the basic problems in elastodynamics. The analyses for transient problems of spherical structures are important and interesting research fields for engineers and scientists. The applications for nonhomogeneous orthotropic hollow sphere have continuously increased in some engineering areas, including aerospace, offshore, infrared detectors, frequency control filters, chemical vessels, information storage devices, and signal processing devices. Accidental failures of rotating sphere due to flexural vibrations have frequently occurred in rotodynamic machinery such as steam turbines and gas turbines. Free vibrations of elastodynamic have many applications in a micropolar porous cubic crystal, poroelastic material [1-3]. Many applications dealing with the elastic bodies and materials, we can only mention a few recent interesting investigations [4-8], the analysis of the dynamic problems of elastic bodies is an important and interesting research field for engineers and scientists. The hollow spheres are frequently used as structural components and their vibration characteristics are obviously important for practical design. Mahmoud et al. [1, 2] discussed the effect of the rotation on plane vibrations in a transversely isotropic infinite hollow cylinder and the effect of the rotation on wave motion through cylindrical bore in a micropolar porous cubic crystal. Mahmoud [3] studied wave propagation in cylindrical poroelastic dry bones. Abd-Alla and Mahmoud [8, 9] solved magnetothermoelastic problem in rotating non-homogeneous orthotropic hollow cylindrical under the hyperbolic heat conduction model and investigated problem of radial vibrations in non-homogeneity isotropic cylinder under influence of initial stress and magnetic field. Influences of rotation, magnetic field, and gravity on Rayleigh waves in a homogeneous orthotropic elastic half space and the solution of electromechanical wave propagation are investigated by Abd-Alla et al. [10-13]. Marin et al. [14, 15] studied porous materials and nonsimple material problems addressed by the Lagrange's identity. Wang [16] studied the elastodynamic solution for an anisotropic hollow sphere. Ding et al. [17, 18] discussed elastodynamic solution of a non-homogeneous orthotropic hollow cylinder, a solution of a non-homogeneous orthotropic cylindrical shell for axisymmetric plane 
strain dynamic thermo elastic problems. Inclusion of arbitrary shape in magnetoelectro-elastic composite materials has been investigated by Wang and Shen [19]. Ding et al. [20] obtained the analytical solution for the axisymmetric plane strain electroelastic dynamics of a non-homogeneous piezoelectric hollow cylinder. Hou and Leung [21] further study the corresponding problem of magnetoelectroelastic hollow cylinders. Buchanan and Liu [22] discussed an analysis of the free vibration of thick-walled isotropic toroidal shells. Yu et al. [23] investigated wave propagation in non-homogeneous magnetoelectroelastic hollow cylinders. Recently, Abd-Alla and Mahmoud [24] discussed analytical solution of wave propagation in non-homogeneous orthotropic rotating elastic media. Abd-Alla et al. [25] studied the effect of the rotation, magnetic field, and initial stress on peristaltic motion of micropolar fluid. Mahmoud [26] investigated wave propagation in piezoelectric hollow cylinder and influence of rotation and generalized magnetothermoelastic on Rayleigh waves in a granular medium under effect of initial stress and gravity field. Sharma et al. [27] studied free vibration analysis of a viscothermoelastic solid sphere. Abd-Alla et al. [28-33] investigated problem of radial and free vibrations in non-homogeneity cylinder under influence of initial stress rotation and magnetic field. Ozsahin and Taskner [34] investigated contact problem for an elastic layer on an elastic half plane. Daouadji et al. [35] studied the Free transverse vibration of the fluid-conveying singlewalled carbon nanotube using nonlocal elastic theory. The present paper deals with the problem of free vibrationsof elastodynamic equations of rotating non-homogeneous and orthotropic hollow sphere. The effect of non-homogeneous and rotation in the equations of motion has been taken into account and the numerical results of the fundamental frequency equations are discussed. Comparisons are made with the result in the present and absence of non-homogeneous and rotation in cases of orthotropic hollow sphere.

\section{Formulation of the Problem}

Take the spherical coordinates $(r, \theta, \varphi)$ and consider elastodynamic problem of non-homogeneous rotating hollow sphere of inner radius $a$ and outer radius $b$, as Figure 1. The stressesstrain relations for non-homogeneous spherically orthotropic material in two dimensions are in the form

$$
\begin{gathered}
\sigma_{r r}=r^{2 m}\left(\alpha_{11} e_{r r}+\alpha_{12} e_{\theta \theta}+\alpha_{13} e_{\varphi \varphi}\right), \\
\sigma_{\theta \theta}=r^{2 m}\left(\alpha_{12} e_{r r}+\alpha_{22} e_{\theta \theta}+\alpha_{23} e_{\varphi \varphi}\right), \\
\sigma_{\varphi \varphi}=r^{2 m}\left(\alpha_{13} e_{r r}+\alpha_{23} e_{\theta \theta}+\alpha_{33} e_{\varphi \varphi}\right), \\
\tau_{r \theta}=r^{2 m} \alpha_{44} e_{r \theta}, \quad \tau_{r \theta}=0, \quad \tau_{\theta \varphi}=0 .
\end{gathered}
$$

The strain-displacements relations in two dimensions are in the form

$$
\begin{aligned}
& e_{r r}=\frac{\partial u_{r}}{\partial r}, \quad e_{\theta \theta}=\frac{1}{r}\left(\frac{\partial u_{\theta}}{\partial \theta}+u_{r}\right), \\
& e_{\varphi \varphi}=\frac{1}{r \sin \theta}\left(u_{r} \sin \theta+u_{\theta} \cos \theta\right),
\end{aligned}
$$

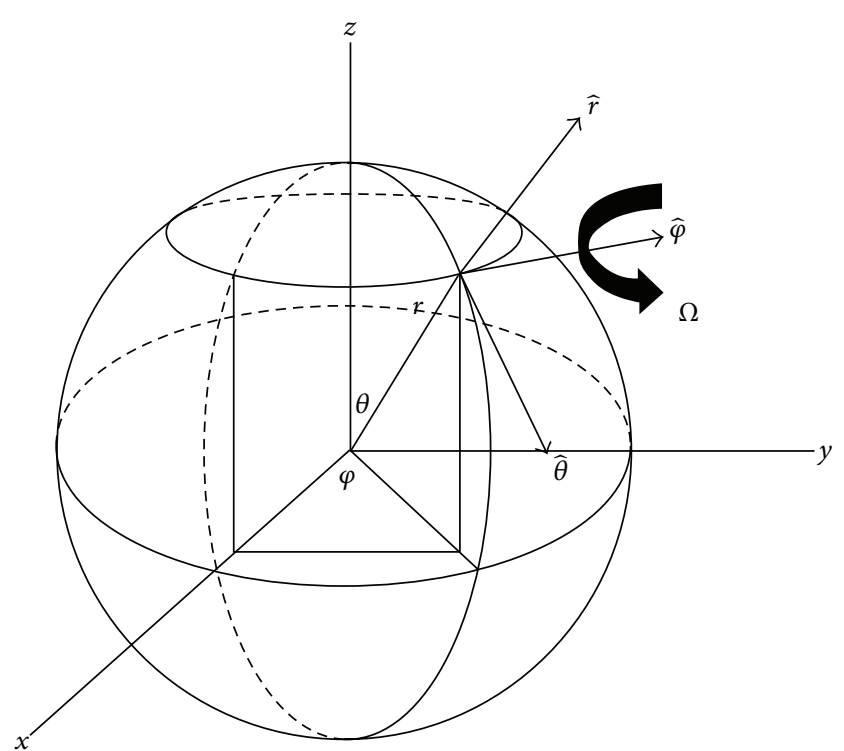

Figure 1: Problem geometry of non-homogeneous orthotropic material.

$$
e_{r \theta}=\frac{1}{2}\left(\frac{1}{r} \frac{\partial u_{r}}{\partial \theta}+\frac{\partial u_{\theta}}{\partial r}-\frac{u_{\theta}}{r}\right), \quad e_{r \varphi}=0, \quad e_{\theta \varphi}=0
$$

Substituting from (1b) into (1a) we obtain the stressesdisplacements relations in two dimensions in the form

$$
\begin{gathered}
\sigma_{r r}=r^{1+2 m}\left(\left(\alpha_{11}+\alpha_{13}\right) u_{r}+\alpha_{13} u_{\theta} \cot \theta\right. \\
\left.+\alpha_{12} \frac{\partial u_{\theta}}{\partial \theta}+r \alpha_{11} \frac{\partial u_{r}}{\partial r}\right), \\
\sigma_{\theta \theta}=r^{1+2 m}\left(\alpha_{22}+\alpha_{22}\right) u_{r}+\alpha_{23} u_{\theta} \cot \theta \\
\left.+\alpha_{22} \frac{\partial u_{\theta}}{\partial \theta}+r \alpha_{12} \frac{\partial u_{r}}{\partial r}\right), \\
\sigma_{\varphi \varphi}=r^{1+2 m}\left(\alpha_{23}+\alpha_{33}\right) u_{r}+\alpha_{33} u_{\theta} \cot \theta \\
\left.+\alpha_{23} \frac{\partial u_{\theta}}{\partial \theta}+r \alpha_{13} \frac{\partial u_{r}}{\partial \theta}\right), \\
\left.\tau_{r \theta}=\frac{\partial u_{r}}{2}+r \frac{\partial u_{\theta}}{\partial r}\right) \\
\tau_{r \theta}^{1+2 m} \alpha_{44}\left(-u_{\theta}+\frac{\tau_{\theta \varphi}}{\partial \theta}=0,\right.
\end{gathered}
$$

where $u_{r}$ and $u_{\theta}$ are, respectively, the components of displacement in the radial and tangential directions, $e_{i j}$ are the strain components, and $\sigma_{i j}$ are the stress components. Where we have characterized the elastic constants $c_{i j}$ and the density $\rho$ of non-homogeneous material in the form

$$
c_{i j}=\alpha_{i j} r^{2 m}, \quad \rho=\rho_{0} r^{2 m}, \quad i, j=1,2,3,
$$


where $\alpha_{i j}$ and $\rho_{0}$ are the values of $c_{i j}$ and $\rho$ in the homogeneous case, respectively, and $m$ is the non-homogeneous parameter. The displacement equations of motion in the rotating frame have two additional terms centripetal acceleration $\vec{\Omega} \times(\vec{\Omega} \times$ $\vec{u})=\left(-\Omega^{2} u_{r},-\Omega^{2} u_{\theta}, 0\right)$, due to time varying motion only, where $\vec{\Omega}=(0,0, \Omega)$, and $\vec{u}=\left(u_{r}, u_{\theta}, 0\right)$.

The elastodynamic equations of rotating non-homogeneous medium in two dimensions in the direction $r, \theta$ are given by:

$$
\begin{aligned}
\frac{\partial \sigma_{r r}}{\partial r} & +\frac{1}{r} \frac{\partial \tau_{r \theta}}{\partial \theta}+\frac{1}{r}\left(2 \sigma_{r r}-\sigma_{\theta \theta}-\sigma_{\varphi \varphi}+\tau_{r \theta} \cot \theta\right) \\
& +\rho_{0} r^{2 m} \Omega^{2} u_{r}=\rho_{0} r^{2 m} \frac{\partial^{2} u_{r}}{\partial t^{2}}, \\
\frac{\partial \tau_{r \theta}}{\partial \theta} & +\frac{1}{r} \frac{\partial \sigma_{\theta \theta}}{\partial \theta}+\frac{1}{r}\left(\left(\sigma_{\theta \theta}-\sigma_{\varphi \varphi}\right) \cot \theta+3 \tau_{r \theta}\right) \\
& +\rho_{0} r^{2 m} \Omega^{2} u_{\theta}=\rho_{0} r^{2 m} \frac{\partial^{2} u_{\theta}}{\partial t^{2}} .
\end{aligned}
$$

Substituting from (1a), (1b), and (2) into (4) and (5), we obtain:

$$
\begin{aligned}
r^{-1+m}\left[2\left(a_{0}+r^{2} \rho_{0} \Omega^{2}\right) u_{r}+a_{1} u_{\theta} \cot \theta+a_{2} \frac{\partial u_{\theta}}{\partial \theta}\right. \\
+\alpha_{44} \cot \theta \frac{\partial u_{r}}{\partial \theta}-\frac{\partial u_{\theta}}{\partial \theta}+\frac{\partial^{2} u_{r}}{\partial \theta^{2}}+r-2 r \rho_{0} \frac{\partial^{2} u_{\theta}}{\partial t^{2}} \\
+4(1+m) \alpha_{11} \frac{\partial u_{r}}{\partial r}+\left(2 \alpha_{13}+\alpha_{44}\right) \cot \theta \frac{\partial u_{\theta}}{\partial r} \\
\left.+\left(2 \alpha_{12}+\alpha_{44}\right) \frac{\partial^{2} u_{\theta}}{\partial r \partial \theta}+2 \alpha_{11} r \frac{\partial^{2} u_{r}}{\partial r^{2}}\right]=0
\end{aligned}
$$

where $a_{0}=\alpha_{12}+2 m \alpha_{12}+\alpha_{13}+2 m \alpha_{13}-\alpha_{22}-2 \alpha_{23}-\alpha_{33}$, $a_{1}=\left((2+4 m) \alpha_{13}-2\left(\alpha_{23}+\alpha_{33}\right)-\alpha_{44}\right)$, and $a_{2}=2\left(\alpha_{12}+\right.$ $\left.2 m \alpha_{12}-\alpha_{22}-\alpha_{23}\right)$.

One has

$$
\begin{aligned}
r^{-1+m}[ & 2\left(\alpha_{22}-\alpha_{33}\right) u_{r} \cot \theta \\
& -2\left(a_{3}-r^{2} \rho_{0} \Omega^{2}+\alpha_{33} \csc ^{2} \theta\right) u_{\theta} \\
& +a_{4} \frac{\partial u_{r}}{\partial \theta}+2 \alpha_{22} \cot \theta \frac{\partial u_{\theta}}{\partial \theta}+\frac{\partial^{2} u_{\theta}}{\partial \theta^{2}} \\
& +r\left(-2 r \rho_{0} \frac{\partial^{2} u_{\theta}}{\partial t^{2}}+2\left(\alpha_{12}-\alpha_{13}\right) \cot \theta \frac{\partial u_{r}}{\partial r}\right. \\
& \left.+2(1+m) \alpha_{44} \frac{\partial u_{\theta}}{\partial r}\right) \\
& \left.+r\left(a_{5} \frac{\partial^{2} u_{r}}{\partial r \partial \theta}+r \alpha_{44} \frac{\partial^{2} u_{\theta}}{\partial \theta^{2}}\right)\right]=0,
\end{aligned}
$$

where $a_{3}=\alpha_{23}-\alpha_{33}+\alpha_{44}+m \alpha_{44}, a_{4}=2\left(\alpha_{22}+\alpha_{23}+\alpha_{44}+m \alpha_{44}\right)$, and $a_{5}=2 \alpha_{12}+\alpha_{44}$.

\section{Solution of the Problem}

By Helmohltz's theorem, the displacement vector $\overleftarrow{u}$ can be written as

$$
\overleftarrow{u}=\underline{\nabla} \Phi_{1}+\underline{\nabla} \wedge \overleftarrow{\Psi}
$$

where the two functions $\Phi_{1}$ and $\overleftarrow{\Psi}$ are known in the theory of elasticity, by Lame' potentials irrotational and rotatoinal parts of the displacement vector $\overleftarrow{u}$, respectively. The displacement potentials are introduced for facilitating the solution of the field equations (5) and (6). It is possible to take only one components of the vector $\overleftarrow{\Psi}$ to be nonzero $\overleftarrow{\Psi}=\left(0,0, \psi_{1}\right)$. From (8), we obtain

$$
\begin{gathered}
u_{r}=\frac{\cot \theta \Psi_{1}+(\partial / \partial \theta) \Psi_{1}}{r}+\frac{\partial \Phi_{1}}{\partial r} \\
u_{\theta}=-\frac{\Psi_{1}-\partial \Phi_{1} / \partial \theta+r(\partial / \partial r) \Psi_{1}}{r} .
\end{gathered}
$$

Substituting from (9) into (6) and (7) and regrouping them lead to the following equations for $\Phi_{1}$ and $\Psi_{1}$ :

$$
\begin{aligned}
r^{-2+m}\left[2\left(a_{1}+r^{2} \rho_{0} \Omega^{2}\right) h_{1}-a_{2} \cot \theta\left(\psi_{1}-\frac{\partial \phi_{1}}{\partial \theta}+r \frac{\partial \psi_{1}}{\partial r}\right)\right. & \\
& -a_{3}\left(\frac{\partial \psi_{1}}{\partial \theta}-\frac{\partial^{2} \phi_{1}}{\partial \theta^{2}}+r \frac{\partial^{2} \psi_{1}}{\partial r \partial \theta}\right) \\
& +\alpha_{44}\left(\cot \theta \csc ^{2} \theta \psi_{1}-\csc ^{2} \theta \frac{\partial \psi_{1}}{\partial \theta}-\frac{\partial^{2} \phi_{1}}{\partial \theta^{2}}\right. \\
& \left.+2 \cot \theta \frac{\partial^{2} \psi_{1}}{\partial \theta^{2}}+\frac{\partial^{3} \psi_{1}}{\partial \theta^{3}}+r h_{2}\right) \\
& -2 \rho_{0} r^{2}\left(\cot \theta \frac{\partial^{2} \psi_{1}}{\partial t^{2}}+\frac{\partial^{3} \psi_{1}}{\partial \theta \partial t^{2}}+r \frac{\partial^{3} \phi_{1}}{\partial r \partial t^{2}}\right) \\
& +4(1+m) \alpha_{11} r^{2} h_{3}+\left(2 \alpha_{13}+\alpha_{44}\right) \\
& \times \cot \theta h_{3}+\left(2 \alpha_{12}+\alpha_{44}\right) h_{4} \\
& \left.+2 \alpha_{11}\left(2 \cot \theta \psi_{1}+2 \frac{\partial \psi_{1}}{\partial \theta}+r h_{5}\right)\right]=0
\end{aligned}
$$

where

$$
\begin{aligned}
& h_{1}=\left(\cot \theta \psi_{1}+\frac{\partial \psi_{1}}{\partial \theta}+r \frac{\partial \phi_{1}}{\partial r}\right), \\
& h_{2}=\left(\cot \theta \frac{\partial^{2} \phi_{1}}{\partial r \partial \theta}+\frac{\partial^{2} \psi_{1}}{\partial r \partial \theta}+\frac{\partial^{3} \phi_{1}}{\partial r \partial \theta^{2}}\right),
\end{aligned}
$$




$$
\begin{gathered}
h_{3}=\left(-\left(\cot \theta \psi_{1}+\left(\frac{\partial \psi_{1}}{\partial \theta}\right)\right.\right. \\
\left.-r\left(\cot \theta\left(\frac{\partial \psi_{1}}{\partial r}\right)+\frac{\partial^{2} \psi_{1}}{\partial r \partial \theta}\right)\right) \\
\left.\times r^{-2}+\frac{\partial^{2} \phi_{1}}{\partial r^{2}}\right), \\
h_{3}=\left(\psi_{1}-\frac{\partial \phi_{1}}{\partial \theta}-r\left(\frac{\partial \psi_{1}}{\partial r}-\frac{\partial^{2} \phi_{1}}{\partial r \partial \theta}+r \frac{\partial^{2} \psi_{1}}{\partial r^{2}}\right)\right), \\
h_{4}=\left(\frac{\partial \psi_{1}}{\partial \theta}-\frac{\partial^{2} \phi_{1}}{\partial \theta^{2}}-r\left(\frac{\partial^{2} \psi_{1}}{\partial r \partial \theta}-\frac{\partial^{3} \phi_{1}}{\partial r \partial \theta^{2}}+r \frac{\partial^{3} \psi_{1}}{\partial r^{2} \partial \theta}\right)\right), \\
\left.\left.+r \cot \theta \frac{\partial^{2} \psi_{1}}{\partial r^{2}}+\frac{\partial^{3} \psi_{1}}{\partial r^{2} \partial \theta}+r \frac{\partial^{3} \phi_{1}}{\partial r^{3}}\right)\right) .
\end{gathered}
$$

One has

$$
\begin{aligned}
& r^{-2+m}\left(2\left(\alpha_{22}-\alpha_{33}\right) \cot \theta\left(\cot \theta \psi_{1}+\frac{\partial \psi_{1}}{\partial \theta}+r \frac{\partial \phi_{1}}{\partial r}\right)\right. \\
& +2\left(a_{3}-r^{2} \rho_{0} \Omega^{2}+\alpha_{33} \csc ^{2} \theta\right)\left(\psi_{1}-\frac{\partial \phi_{1}}{\partial \theta}+r \frac{\partial \psi_{1}}{\partial r}\right) \\
& +2\left(a_{6}\left(-\csc ^{2} \theta \psi_{1}+\cot \theta \frac{\partial \psi_{1}}{\partial \theta}+\frac{\partial^{2} \psi_{1}}{\partial \theta^{2}}+r \frac{\partial^{2} \phi_{1}}{\partial r \partial \theta}\right)\right. \\
& -\alpha_{22}\left(\frac{\partial^{2} \psi_{1}}{\partial \theta^{2}}-\frac{\partial^{3} \phi_{1}}{\partial \theta^{3}}\right. \\
& +\cot \theta\left(\frac{\partial \psi_{1}}{\partial \theta}-\frac{\partial^{2} \phi_{1}}{\partial \theta^{2}}+r \frac{\partial^{2} \psi_{1}}{\partial r \partial \theta}\right) \\
& \left.\left.+r \frac{\partial^{3} \psi_{1}}{\partial r \partial \theta^{2}}\right)\right) \\
& +r^{2}\left(2 \rho_{0}\left(\frac{\partial^{2} \psi_{1}}{\partial t^{2}}-\frac{\partial^{3} \phi_{1}}{\partial \theta \partial t^{2}}+r \frac{\partial^{3} \psi_{1}}{\partial r \partial t^{2}}\right)\right. \\
& -\alpha_{44}\left(\frac{\partial^{2} \psi_{1}}{\partial \theta^{2}}-\frac{\partial^{3} \phi_{1}}{\partial \theta^{3}}+r \frac{\partial^{3} \psi_{1}}{\partial r \partial \theta^{2}}\right) \\
& +2\left(\alpha_{12}-\alpha_{13}\right) \\
& \times \cot \theta\left(-\frac{h_{8}}{r^{2}}+\frac{\partial^{2} \phi_{1}}{\partial r^{2}}\right)-\frac{2(1+m) \alpha_{44} h_{7}}{r^{2}} \\
& \left.\left.+\frac{\left(2 \alpha_{12}+\alpha_{44}\right) h_{6}}{r^{2}}\right)\right)=0 \text {, }
\end{aligned}
$$

where

$$
\begin{gathered}
a_{6}=\left(\alpha_{22}+\alpha_{23}+\alpha_{44}+m \alpha_{44}\right), \\
h_{6}=\left(-\cot \theta \frac{\partial \psi_{1}}{\partial \theta}-\frac{\partial^{2} \psi_{1}}{\partial \theta^{2}}+\csc ^{2} \theta\left(\psi_{1}-r \frac{\partial \psi_{1}}{\partial r}\right)\right. \\
\left.+r \cot \theta \frac{\partial^{2} \psi_{1}}{\partial r \partial \theta}+r \frac{\partial^{3} \psi_{1}}{\partial r \partial \theta^{2}}\left(r \frac{\partial^{3} \phi_{1}}{\partial r^{2} \partial \theta}\right)\right), \\
h_{7}=\left(-\psi_{1}+\frac{\partial \phi_{1}}{\partial \theta}+r\left(\frac{\partial \psi_{1}}{\partial r}-\frac{\partial^{2} \phi_{1}}{\partial r \partial \theta}+r \frac{\partial^{2} \psi_{1}}{\partial r^{2}}\right)\right), \\
h_{8}=\cot \theta \psi_{1}+\frac{\partial \psi_{1}}{\partial \theta}-r\left(\cot \theta \frac{\partial \psi_{1}}{\partial r}+\frac{\partial^{2} \psi_{1}}{\partial r \partial \theta}\right) .
\end{gathered}
$$

To study the propagation of harmonic waves, we assume a solution in the form

$$
\begin{aligned}
& \Phi_{1}(r, \theta, t)=\Phi_{2}(r) e^{i(\gamma \theta-\omega t)}, \\
& \Psi_{1}(r, \theta, t)=\Psi_{2}(r) e^{i(\gamma \theta-\omega t)} .
\end{aligned}
$$

Substituting from (14) into (10) and (12) and regrouping them lead to the following equations for $\Phi_{2}$ and $\Psi_{2}$ :

$$
\begin{aligned}
& e^{i(\gamma \theta-t \omega)} r^{-1+m}\left[2 \gamma\left(a_{7}-a_{8} \cot \theta\right) \phi_{2}\right. \\
& +\left(-i \gamma\left(a_{9}-2 r^{2} \rho_{0}\left(\omega^{2}+\Omega^{2}\right)\right.\right. \\
& \left.+\alpha_{44} \csc ^{2} \theta\right) \\
& +\cot \theta\left(2\left(a_{10}+r^{2} \rho_{0}\left(\omega^{2}+\Omega^{2}\right)\right)\right. \\
& \left.\left.+\alpha_{44} \csc ^{2} \theta\right)\right) \Psi_{2} \\
& +r\left(2 \left(a_{11}+r^{2} \rho_{0}\left(\omega^{2}+\Omega^{2}\right)\right.\right. \\
& \left.+i\left(a_{13}+\alpha_{44}\right) \gamma \cot \theta\right) \frac{d \Phi_{2}}{d r} \\
& +2\left(a_{12}+a_{13} \cot \theta\right) \frac{d \Psi_{2}}{d r} \\
& +r\left(4(1+m) a_{11} \frac{d^{2} \Phi_{2}}{d r^{2}}\right. \\
& +\left(a_{14}+a_{15} \cot \theta\right) \frac{d^{2} \Psi_{2}}{d r^{2}} \\
& \left.\left.\left.+2 r \alpha_{11} \frac{d^{3} \Phi_{2}}{d r^{3}}\right)\right)\right]=0
\end{aligned}
$$


where

$$
\begin{aligned}
a_{7}= & \left(-2 m \alpha_{12}+\alpha_{22}+\alpha_{23}+\alpha_{44}\right) \gamma \\
a_{8}= & i\left(-2 m \alpha_{13}+\alpha_{23}+\alpha_{33}+\alpha_{44}\right) \\
a_{9}= & -2 \alpha_{12}+4 m\left(\alpha_{11}-\alpha_{13}\right)-2 \alpha_{13}+2 \alpha_{23} \\
& +2 \alpha_{33}+\alpha_{44}\left(-1+\gamma^{2}\right) \\
a_{10}= & \alpha_{12}+2 m\left(-\alpha_{11}+\alpha_{12}\right)+\alpha_{13}-\alpha_{22} \\
& -\alpha_{23}+\alpha_{44}-\alpha_{44} \gamma^{2}, \\
a_{11}= & \alpha_{12}+2 m \alpha_{12}+\alpha_{13}+2 m \alpha 13-\alpha_{22} \\
& -2 \alpha_{23}-\alpha_{33}-\left(\alpha_{12}+\alpha_{44}\right) \gamma^{2}, \\
a_{12}= & i\left(2 m \alpha_{11}-2 \alpha_{12}-2 m \alpha_{12}+\alpha_{22}+\alpha_{23}\right) \gamma, \\
a_{13}= & 2\left(2 m \alpha_{11}-2 \alpha_{13}-2 m \alpha_{13}+\alpha_{23}+\alpha_{33}\right), \\
a_{14}= & i\left(2 \alpha_{11}-2 m \alpha_{12}-\alpha_{44}\right) \gamma+\left(2 \alpha_{11}-2 \alpha_{13}-\alpha_{44}\right), \\
a_{15}= & \left(2 m \alpha_{11}-2 \alpha_{13}-\alpha_{44}\right) . \\
&
\end{aligned}
$$

One has

$$
\begin{aligned}
& e^{i(\gamma \theta-t \omega)} r^{-1+m}\left[-i \gamma\left(a_{16}+\alpha_{44}\left(4+4 m+r^{2} \gamma^{2}\right)\right.\right. \\
& -2 r^{2} \rho_{0}\left(\omega^{2}+\Omega^{2}\right)-2 i \alpha_{22} \gamma \cot \theta \\
& \left.+2 \alpha 33 \csc ^{2} \theta\right) \Phi_{2}+2\left(\alpha_{12}-\alpha_{13}\right) \Psi_{2} \\
& +\left(a_{17}+\left(-1-2 m+r^{2}\right) \alpha_{44} \gamma^{2}\right. \\
& -2 \alpha_{23}\left(-1+\gamma^{2}\right)-2 r^{2} \rho_{0}\left(\omega^{2}+\Omega^{2}\right) \\
& \left.-a_{18} \cot \theta+a_{19} \csc ^{2} \theta\right) \Psi_{2} \\
& +r\left(2 i\left(a_{20}-i\left(\alpha_{22}-\alpha_{23}\right) \cot \theta\right) \frac{d \Phi_{2}}{d r}\right. \\
& -\left(a_{21}+2 r^{2} \rho_{0} \omega^{2}+2 r^{2} \rho_{0} \Omega^{2}\right. \\
& \left.-a_{22} \cot \theta+a_{23} \csc ^{2} \theta\right) \frac{d \Psi_{2}}{d r} \\
& +r\left(i\left(2 \alpha_{12}+\alpha_{44}\right) \gamma\right. \\
& \left.+2\left(\alpha_{12}-\alpha_{13}\right) \cot \theta\right) \frac{d^{2} \Phi_{2}}{d r^{2}} \\
& \left.\left.-2(1+m) \alpha_{44} r \frac{d^{2} \Psi_{2}}{d r^{2}}\right)\right]=0,
\end{aligned}
$$

where

$$
\begin{aligned}
a_{16}= & 2 \alpha_{23}-2 \alpha_{33}+2 \alpha_{22} \gamma^{2}, \\
a_{17}= & -2 \alpha_{22}+4(1+m) \alpha_{44}+2 \alpha_{12} \gamma^{2}, \\
a_{18}= & i\left(4 \alpha_{12}-2\left(\alpha_{13}+\alpha_{22}+\alpha_{23}-\alpha_{33}\right)\right. \\
& \left.-(1+2 m) \alpha_{44}\right) \gamma \\
a_{19}= & \left(2 \alpha_{13}-2 \alpha_{23}-\alpha_{44}-2 m \alpha_{44}\right), \\
a_{20}= & \left(\alpha_{22}+\alpha_{23}+2(1+m) \alpha_{44}\right) \gamma, \\
a_{21}= & -2\left(\alpha_{13}+\alpha_{23}-\alpha_{33}\right)-2 \alpha_{22} \gamma^{2} \\
& +\alpha_{44} \gamma^{2}-r^{2} \alpha_{44} \gamma^{2}+2 \alpha_{12}\left(1+\gamma^{2}\right), \\
a_{22}= & i\left(4 \alpha_{12}-2\left(\alpha_{13}+\alpha_{22}\right)+\alpha_{44}\right) \gamma, \\
a_{23}= & \left(2 \alpha_{13}-2 \alpha_{33}+\alpha_{44}\right),
\end{aligned}
$$

where $\gamma$ is the wave number, $\omega$ is the angular frequency, $\gamma=$ $2 \pi / \lambda$, and $\lambda$ is the wavelength. Substituting from (14) into (15) and (17) and after regrouping them leads to two independent equations for $\Phi_{2}$ and $\Psi_{2}$; these equations are called spherical Bessel's equations whose general solution is in the form

$$
\begin{gathered}
\Phi_{2}(r)=A_{1} j_{n}(k r)+A_{2} y_{n}(k r), \\
\Psi_{2}(r)=A_{3} j_{n}\left(k_{1} r\right)+A_{4} y_{n}\left(k_{1} r\right),
\end{gathered}
$$

where

$$
\begin{gathered}
n(n+1)=\frac{\left(\alpha_{22}+\alpha_{33}+2 \alpha_{23}\right)-(2 m+1)\left(\alpha_{12}+\alpha_{13}\right)}{\alpha_{11}} \\
+m(m+1), \\
k^{2}=\frac{\alpha_{44}+\rho_{0} \omega^{2}}{\alpha_{11}}+L_{1}-\gamma^{2}\left(\alpha_{11}-2 \alpha_{43}\right)+\frac{\rho_{0}}{\alpha_{11}}\left(\Omega^{2}+\omega^{2}\right), \\
k_{1}^{2}=\frac{\rho_{0}}{\alpha_{11}}\left(\Omega^{2}+\omega^{2}\right)+\frac{\left(L_{2}+\rho_{0} \omega^{2}\right)}{\alpha_{11}} \\
\quad+2 \gamma^{2}\left(\alpha_{12}+2 m \alpha_{12}-\alpha_{22}-\alpha_{23}\right), \\
L_{1}=\left((2+4 m) \alpha_{13}-2\left(\alpha_{23}+\alpha_{33}\right)-m \alpha_{44}\right), \\
L_{2}=2 \alpha_{12}+2 m \alpha_{13}-\alpha_{22}-2 \alpha m_{23}-\alpha_{33},
\end{gathered}
$$

where $A_{1}, A_{2}, A_{3}$, and $A_{4}$ are arbitrary constants and $j_{n}(k r)$ and $y_{n}(k r)$ denote spherical Bessel's functions of the first and second kind of order $n$, respectively, which are defined in terms of Bessel's function as follows: $j_{n}(k r)=$ $\sqrt{\pi / 2 k r} J_{n+1 / 2}(k r), y_{n}(k r)=\sqrt{\pi / 2 k r} Y_{n+1 / 2}(k r)$. From (19) and (14) we get the following solutions for $\Phi_{1}$ and $\Psi_{1}$ as follows:

$$
\begin{gathered}
\Phi_{1}(r, \theta, t)=e^{i(\gamma \theta-w t)}\left[A_{1} j_{n}(k r)+A_{2} y_{n}(k r)\right], \\
\Psi_{1}(r, \theta, t)=e^{i(\gamma \theta-w t)}\left[A_{3} j_{n}\left(k_{1} r\right)+A_{4} y_{n}\left(k_{1} r\right)\right] .
\end{gathered}
$$


Substituting from (21) into (9), we obtain the final solution of the displacement components in the following form:

$$
\begin{aligned}
u_{r}=\frac{1}{r} e^{i(\gamma \theta-w t)}[ & A_{1}\left\{n j_{n}(k r)-r k j_{n+1}(k r)\right\} \\
& +A_{2}\left\{n y_{n+1}(k r)+y_{n+1}(k r)\right\} \\
& \left.+A_{3}(i \gamma+\cot \theta) j_{n}\left(k_{1} r\right)+A_{4} y_{n+1}\left(k_{1} r\right)\right], \\
u_{\theta}=\frac{1}{r} e^{i(\gamma \theta-w t)}[ & A_{1} i \gamma j_{n}(k r)+A_{2} y_{n}(k r) \\
& -A_{3}\left\{(1+n) j_{n}\left(k_{1} r\right)+k_{1} r j_{n+1}\left(k_{1} r\right)\right\} \\
& \left.-A_{4}\left\{(1+n) y_{n}\left(k_{1} r\right)+r k_{1} y_{n+1}\left(k_{1} r\right)\right\}\right] .
\end{aligned}
$$

Substituting from (22) into (2), we obtain the final solution of the stress components in the following form:

$$
\begin{aligned}
& \sigma_{r r}=r^{-2+2 m} e^{i(\gamma \theta-t \omega)} \\
& \times\left[A _ { 1 } \left\{\left(\left(\alpha_{12}+\alpha_{13}\right) n\right.\right.\right. \\
& +\alpha_{11}\left((-1+n) n-k^{2} r^{2}\right)-\alpha_{12} \gamma^{2} \\
& \left.+i \alpha_{13} \gamma \cot \theta\right) j_{n}(k r) \\
& \left.+\left(2 \alpha_{11}-\alpha_{12}-\alpha_{13}\right) k r j_{n+1}(k r)\right\} \\
& +A_{3}\left\{\left(i\left(\alpha_{13}+\alpha_{11}(-1+n)-\alpha_{12} n\right) \gamma\right.\right. \\
& +\left(\alpha_{12}+\alpha_{11}(-1+n)\right. \\
& \left.\left.-\alpha_{13} n\right) \cot \theta\right) j_{n}\left(k_{1} r\right) \\
& +k_{1} r\left(-i\left(\alpha_{11}-\alpha_{12}\right) \gamma+\left(-\alpha_{11}+\alpha_{13}\right)\right. \\
& \left.\times \cot \theta) j_{n+1}\left(k_{1} r\right)\right\} \\
& +A_{2}\left\{\left(\left(\alpha_{12}+\alpha_{13}\right) n+\alpha_{11}\left((-1+n) n-k^{2} r^{2}\right)\right.\right. \\
& \left.-\alpha_{12} \gamma^{2}+i \alpha_{13} \gamma \cot \theta\right) y_{n}(k r) \\
& \left.+\left(2 \alpha_{11}-\alpha_{12}-\alpha_{13}\right) k r y_{n+1}(k r)\right\} \\
& +A_{4}\left\{\left(i\left(\alpha_{13}+\alpha_{11}(-1+n)-\alpha_{12} n\right) \gamma\right.\right. \\
& +\left(\alpha_{12}+\alpha_{11}(-1+n)\right. \\
& \left.\left.-\alpha_{13} n\right) \cot \theta\right) y_{n}\left(k_{1} r\right) \\
& +k_{1} r\left(-i\left(\alpha_{11}-\alpha_{12}\right) \gamma\right. \\
& \left.\left.\left.+\left(-\alpha_{11}+\alpha_{13}\right) \cot \theta\right) y_{n+1}\left(k_{1} r\right)\right\}\right],
\end{aligned}
$$

$\sigma_{\theta \theta}=r^{-2+2 m} e^{i(\gamma \theta-t \omega)}$

$\times\left[A_{1}\left\{\left(\left(\alpha_{22}+\alpha_{23}\right) n\right.\right.\right.$

$$
+\alpha_{12}\left((-1+n) n-k^{2} r^{2}\right)-\alpha_{22} \gamma^{2}
$$$$
\left.+i \alpha_{23} \gamma \cot \theta\right) j_{n}(k r)
$$$$
\left.+\left(2 \alpha_{12}-\alpha_{22}-\alpha_{23}\right) k r j_{n+1}(k r)\right\}
$$$$
+A_{3}\left\{\left(i\left(\alpha_{23}+\alpha_{12}(-1+n)-\alpha_{22} n\right) \gamma\right.\right.
$$$$
+\left(\alpha_{22}+\alpha_{12}(-1+n)\right.
$$$$
\left.\left.-\alpha_{23} n\right) \cot \theta\right) j_{n}\left(k_{1} r\right)
$$$$
+k_{1} r\left(-i\left(\alpha_{12}-\alpha_{22}\right) \gamma+\left(-\alpha_{12}+\alpha_{23}\right)\right.
$$$$
\left.\times \cot \theta) j_{n+1}\left(k_{1} r\right)\right\}
$$

$$
+A_{2}\left\{\left(\left(\alpha_{22}+\alpha_{23}\right) n+\alpha_{12}\left((-1+n) n-k^{2} r^{2}\right)\right.\right.
$$$$
\left.-\alpha_{22} \gamma^{2}+i \alpha_{23} \gamma \cot \theta\right) y_{n}(k r)
$$$$
\left.+\left(2 \alpha_{12}-\alpha_{22}-\alpha_{23}\right) k r y_{n+1}(k r)\right\}
$$$$
+A_{4}\left\{\left(i\left(\alpha_{23}+\alpha_{12}(-1+n)-\alpha_{22} n\right) \gamma\right.\right.
$$$$
+\left(\alpha_{22}+\alpha_{12}(-1+n)\right.
$$$$
\left.\left.-\alpha_{23} n\right) \cot \theta\right) y_{n+1}\left(k_{1} r\right)
$$

$$
+k_{1} r\left(-i\left(\alpha_{12}-\alpha_{22}\right) \gamma\right.
$$$$
\left.\left.\left.+\left(-\alpha_{12}+\alpha_{23}\right) \cot \theta\right) y_{n+1}\left(k_{1} r\right)\right\}\right] \text {, }
$$

$$
\begin{aligned}
& \sigma_{\varphi \varphi}=r^{-2+2 m} e^{i(\gamma \theta-t \omega)} \\
& \times\left[A_{1}\{(\right.\left(\alpha_{23}+\alpha_{33}\right) n \\
&+ \alpha_{13}\left((-1+n) n-k^{2} r^{2}\right)-\alpha_{23} \gamma^{2} \\
&\left.+i \alpha_{33} \gamma \cot \theta\right) j_{n}(k r) \\
&\left.+\left(2 \alpha_{13}-\alpha_{23}-\alpha_{33}\right) k r j_{n+1}(k r)\right\} \\
&+A_{3}\{( i\left(\alpha_{33}+\alpha_{13}(-1+n)-\alpha_{23} n\right) \gamma \\
&+\left(\alpha_{23}+\alpha_{13}(-1+n)\right. \\
&\left.\left.-\alpha_{33} n\right) \cot \theta\right) j_{n}\left(k_{1} r\right) \\
&+k_{1} r\left(-i\left(\alpha_{13}-\alpha_{23}\right) \gamma+\left(-\alpha_{13}+\alpha_{33}\right)\right. \\
&\left.\times \cot \theta) j_{n+1}\left(k_{1} r\right)\right\} \\
&+A_{2}\{\left(\left(\alpha_{23}+\alpha_{33}\right) n+\alpha_{13}\left((-1+n) n-k^{2} r^{2}\right)\right. \\
&\left.-\alpha_{23} \gamma^{2}+i \alpha_{33} \gamma \cot \theta\right) y_{n}(k r) \\
&+\left.\left(2 \alpha_{13}-\alpha_{23}-\alpha_{33}\right) k r y_{n+1}(k r)\right\} \\
&+A_{4}\{(\left(\alpha_{33}+\alpha_{13}(-1+n)-\alpha_{23} n\right) \gamma \\
&+\left(\alpha_{23}+\alpha_{13}(-1+n)\right. \\
&\left.\left.-\alpha_{33} n\right) \cot \theta\right) y_{n}\left(k_{1} r\right) \\
&+ k_{1} r\left(-i\left(\alpha_{13}-\alpha_{23}\right) \gamma\right. \\
&\left.\left.\left.+\left(-\alpha_{13}+\alpha_{33}\right) \cot \theta\right) y_{n+1}\left(k_{1} r\right)\right\}\right]
\end{aligned}
$$




$$
\begin{aligned}
& \tau_{r \theta}=- \frac{r^{-2+2 m}}{2} \alpha_{44} e^{i(\gamma \theta-t \omega)} \\
& \times\left[A_{1}\left\{-2 i(-1+n) \gamma j_{n}(k r)+2 i k r \gamma j_{n+1}(k r)\right\}\right. \\
&+A_{3}\left\{\left(-2+(-1+n) n-k_{1}^{2} r^{2}+\gamma^{2}-i \gamma \cot \theta\right.\right. \\
&\left.\left.+\csc ^{2} \theta\right) j_{n}\left(k_{1} r\right)+2 k_{1} r j_{n+1}\left(k_{1} r\right)\right\} \\
&-A_{2}\left\{2 i(-1+n) \gamma y_{n}(k r)+2 i k r \gamma y_{n+1}(k r)\right\} \\
&+A_{4}\left\{\left(-2+(-1+n) n-k_{1}^{2} r^{2}+\gamma^{2}-i \gamma \cot \theta\right.\right. \\
&\left.\left.\left.+\csc ^{2} \theta\right) y_{n}\left(k_{1} r\right)+2 k_{1} r y_{n+1}\left(k_{1} r\right)\right\}\right] .
\end{aligned}
$$

From the solutions of elastic wave equations, the systems of equations depend on non-homogenity, rotation and the frequency.

\section{Boundary Conditions and Frequency Equation}

The solutions of the hollow sphere with different boundary conditions are performed, the mixed boundary conditions which consist of two kinds of boundary conditions, the inner surface fixed and the outer surface free, that is,

$$
u_{r}=u_{\theta}=0, \quad r=a, \quad \sigma_{r r}=\tau_{r \theta}=0, \quad r=b .
$$

In this case, from (22), (23a), (23b), (23c), (23d), and (24) we have

$$
\begin{gathered}
A_{1}\left\{n j_{n}(k a)-a k j_{n+1}(k a)\right\} \\
+A_{2}\left\{n y_{n+1}(k a)+y_{n+1}(k a)\right\} \\
+A_{3}(i \gamma+\cot \theta) j_{n}\left(k_{1} a\right)+A_{4} y_{n+1}\left(k_{1} a\right)=0, \\
A_{1} i \gamma j_{n}(k a)+A_{2} y_{n}(k a) \\
-A_{3}\left\{(1+n) j_{n}\left(k_{1} a\right)+k_{1} a j_{n+1}\left(k_{1} a\right)\right\} \\
-A_{4}\left\{(1+n) y_{n}\left(k_{1} a\right)+a k_{1} y_{n+1}\left(k_{1} a\right)\right\}=0, \\
A_{1}\left\{\left(\left(\alpha_{12}+\alpha_{13}\right) n+\alpha_{11}\left((-1+n) n-k^{2} b^{2}\right)\right.\right. \\
\left.\quad-\alpha_{12} \gamma^{2}+i \alpha_{13} \gamma \cot \theta\right) j_{n}(k b) \\
\left.+\left(2 \alpha_{11}-\alpha_{12}-\alpha_{13}\right) k b j_{n+1}(k b)\right\} \\
+A_{2}\left\{\left(\left(\alpha_{12}+\alpha_{13}\right) n+\alpha_{11}\left((-1+n) n-k^{2} b^{2}\right)\right.\right. \\
\left.\quad-\alpha_{12} \gamma^{2}+i \alpha_{13} \gamma \cot \theta\right) y_{n}(k b) \\
\left.\quad+\left(2 \alpha_{11}-\alpha_{12}-\alpha_{13}\right) k b y_{n+1}(k b)\right\}
\end{gathered}
$$

$$
\begin{gathered}
+A_{3}\left\{\left(i\left(\alpha_{13}+\alpha_{11}(-1+n)-\alpha_{12} n\right) \gamma\right.\right. \\
+\left(\alpha_{12}+\alpha_{11}(-1+n)-\alpha_{13} n\right) \\
\times \cot \theta) j_{n}\left(k_{1} b\right) \\
+k_{1} b\left(-i\left(\alpha_{11}-\alpha_{12}\right) \gamma+\left(-\alpha_{11}+\alpha_{13}\right)\right. \\
\left.\quad \times \cot \theta) j_{n+1}\left(k_{1} b\right)\right\} \\
+A_{4}\left\{\left(i\left(\alpha_{13}+\alpha_{11}(-1+n)-\alpha_{12} n\right) \gamma\right.\right. \\
+\left(\alpha_{12}+\alpha_{11}(-1+n)-\alpha_{13} n\right) \\
\times \cot \theta) y_{n}\left(k_{1} b\right) \\
+k_{1} b\left(-i\left(\alpha_{11}-\alpha_{12}\right) \gamma+\left(-\alpha_{11}+\alpha_{13}\right)\right. \\
\left.\times \cot \theta) y_{n+1}\left(k_{1} b\right)\right\}=0,
\end{gathered}
$$

$$
\begin{aligned}
A_{1}\left\{-2 i(-1+n) \gamma j_{n}(k b)+2 i k b \gamma j_{n+1}(k b)\right\} \\
-A_{2}\left\{2 i(-1+n) \gamma y_{n}(k b)+2 i k b \gamma y_{n+1}(k b)\right\} \\
+A_{3}\left\{\left(-2+(-1+n) n-k_{1}^{2} b^{2}+\gamma^{2}-i \gamma \cot \theta\right.\right. \\
\left.\left.+\csc ^{2} \theta\right) j_{n}\left(k_{1} b\right)+2 k_{1} b j_{n+1}\left(k_{1} b\right)\right\} \\
+A_{4}\left\{\left(-2+(-1+n) n-k_{1}^{2} b^{2}+\gamma^{2}-i \gamma \cot \theta\right.\right. \\
\left.\left.+\csc ^{2} \theta\right) y_{n}\left(k_{1} b\right)+2 k_{1} b y_{n+1}\left(k_{1} b\right)\right\} .
\end{aligned}
$$

From (25a), (25b), (25c), and (25d) we get the following frequency equation:

$$
\left|a_{i j}\right|=0, \quad i, j=1,2,3,4,
$$

where the coefficients $a_{i j}$ are functions of rotation, nonhomogenity, frequency, the radius $r$. Finally, we confined our attention to make these quantities dimensionless to simplify the calculation of the eigenvalues of equations. The coefficients $a_{i j}$ are

$$
\begin{aligned}
& a_{11}= n j_{n}(k a)-r k j_{n+1}(k a), \\
& a_{12}= n y_{n+1}(k a)+y_{n+1}(k a), \\
& a_{13}=(i \gamma+\cot \theta) j_{n}\left(k_{1} a\right), \\
& a_{14}= y_{n+1}\left(k_{1} a\right), \quad a_{21}=i \gamma j_{n}(k a), \\
& a_{22}= y_{n}(k a), \\
& a_{23}=(1+n) j_{n}\left(k_{1} a\right)-k_{1} a j_{n+1}\left(k_{1} a\right), \\
& a_{24}=(1+n) y_{n}\left(k_{1} a\right)+a k_{1} y_{n+1}\left(k_{1} a\right), \\
& a_{31}=\left(\left(\alpha_{12}+\alpha_{13}\right) n+\alpha_{11}\left((-1+n) n-k^{2} b^{2}\right)\right. \\
&\left.\quad-\alpha_{12} \gamma^{2}+i \alpha_{13} \gamma \cot \theta\right) j_{n}(k b) \\
& \quad+\left(2 \alpha_{11}-\alpha_{12}-\alpha_{13}\right) k b j_{n+1}(k b),
\end{aligned}
$$




$$
\begin{aligned}
& a_{32}=\left(\left(\alpha_{12}+\alpha_{13}\right) n+\alpha_{11}\left((-1+n) n-k^{2} b^{2}\right)\right. \\
& \left.-\alpha_{12} \gamma^{2}+i \alpha_{13} \gamma \cot \theta\right) y_{n}(k b) \\
& +\left(2 \alpha_{11}-\alpha_{12}-\alpha_{13}\right) k b y_{n+1}(k b), \\
& a_{33}=\left\{\left(i\left(\alpha_{13}+\alpha_{11}(-1+n)-\alpha_{12} n\right) \gamma\right.\right. \\
& +\left(\alpha_{12}+\alpha_{11}(-1+n)-\alpha_{13} n\right) \\
& \times \cot \theta) j_{n}\left(k_{1} b\right) \\
& +k_{1} b\left(-i\left(\alpha_{11}-\alpha_{12}\right) \gamma+\left(-\alpha_{11}+\alpha_{13}\right)\right. \\
& \left.\times \cot \theta) j_{n+1}\left(k_{1} b\right)\right\}, \\
& a_{34}=\left(i\left(\alpha_{13}+\alpha_{11}(-1+n)-\alpha_{12} n\right) \gamma\right. \\
& +\left(\alpha_{12}+\alpha_{11}(-1+n)-\alpha_{13} n\right) \\
& \times \cot \theta) y_{n}\left(k_{1} b\right) \\
& +k_{1} b\left(-i\left(\alpha_{11}-\alpha_{12}\right) \gamma\right. \\
& \left.+\left(-\alpha_{11}+\alpha_{13}\right) \cot \theta\right) y_{n 1+1}\left(k_{1} b\right), \\
& a_{41}=-2 i(-1+n) \gamma j_{n}(k b)+2 i k b \gamma j_{n+1}(k b) \text {, } \\
& a_{42}=-2 i(-1+n) \gamma y_{n}(k b)-2 i k b \gamma y_{n+1}(k b) \text {, } \\
& a_{43}=\left(-2+(-1+n) n-k_{1}^{2} b^{2}+\gamma^{2}\right. \\
& \left.-i \gamma \cot \theta+\csc ^{2} \theta\right) j_{n}\left(k_{1} b\right) \\
& +2 k_{1} b j_{n+1}\left(k_{1} b\right) \text {, } \\
& a_{44}=\left(-2+(-1+n) n-k_{1}^{2} b^{2}+\gamma^{2}\right. \\
& \left.-i \gamma \cot \theta+\csc ^{2} \theta\right) y_{n}\left(k_{1} b\right) \\
& +2 k_{1} b y_{n+1}\left(k_{1} b\right) \text {. }
\end{aligned}
$$

\section{Numerical Results and Discussion}

To examine the influence of non-homogenity, rotation and variation of the non-dimensional frequency in hollow sphere with the radius $r$ have been shown graphically. Free vibrations have been studied using a half-interval method. The frequency equations have been obtained under the effects of non-homogenity and rotation. It is found that the nondimensional frequency increases with the increases of radius $r$ for all cases. As an illustrative example, the elastic constants for an orthotropic non-homogeneous material from Hearmon $[36,37]$ are $\alpha_{23}=3.945, \alpha_{11}=3.198, \alpha_{33}=2.7951$, $\alpha_{13}=2.310, \alpha_{12}=0.713, \alpha_{22}=4.560$, and $\rho=2.680$. Numerical calculations are carried out for the displacement and the stress components along the $r$-direction at different values of the rotation $\Omega=0.0,1.3,2.6$ in the case of nonhomogeneous material and orthotropic material. Figure 2

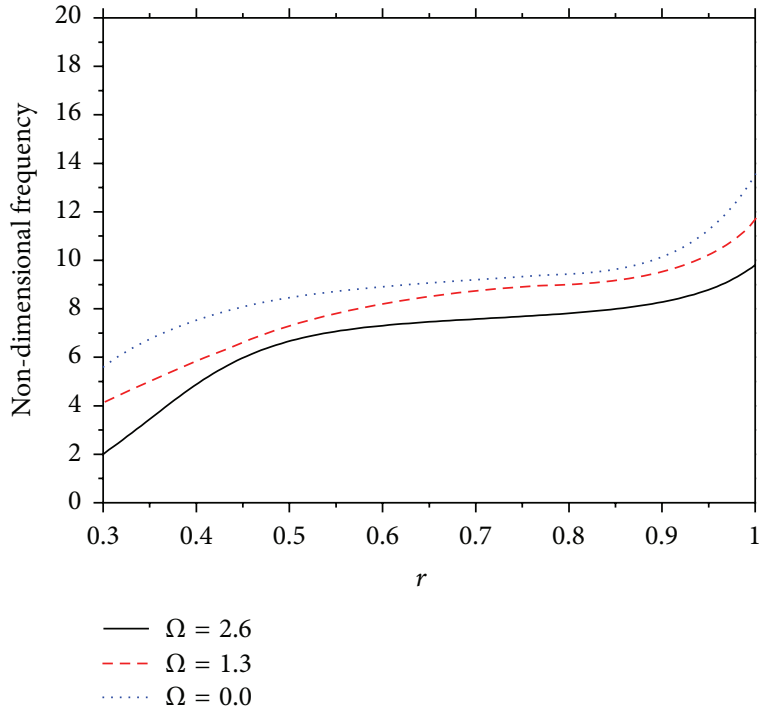

FIGURE 2: Variation of non-dimensional frequency versus the radius $r$, for the various values of rotation $\Omega$ and non-homogeneous $m=$ 0.65 (inner fixed surfaces and outer free surfaces) and $n=0$.

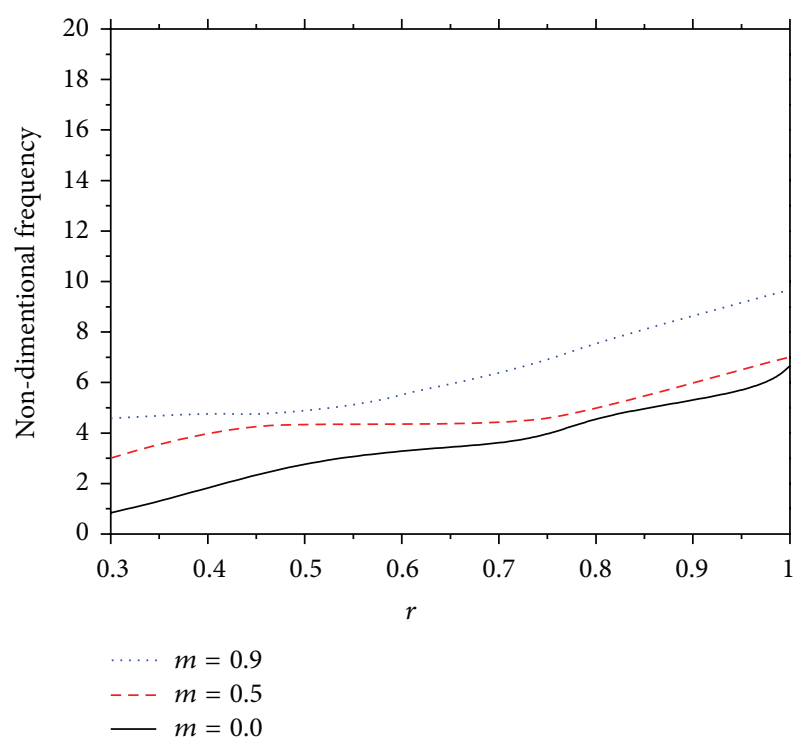

FIGURE 3: Variation of non-dimensional frequency versus the radius $r$, for the various values of non-homogeneous $m$ and rotation $\Omega=$ 2.5 (inner fixed surfaces and outer free surfaces) and $n=0$.

shows the response histories of the non-dimensional frequencies with the radius $r$ for rotating hollow sphere $\Omega=2.5$. With the effect of various values of non-homogeneous $m=$ $0.0,0.5,0.9$ in the case of orthotropic material, it can be found that the distribution of the non-dimensional frequencies is increasing as the increase in the radius $r$ and the nondimensional frequencies are increasing with the increase in the non-homogenity. Figure 3 shows the variation of the nondimensional frequencies with the radius $r$ for hollow sphere with the effect of various values of rotation $\Omega=0.0,1.3,2.6$ in the case of non-homogeneous $m=0.65$ orthotropic 


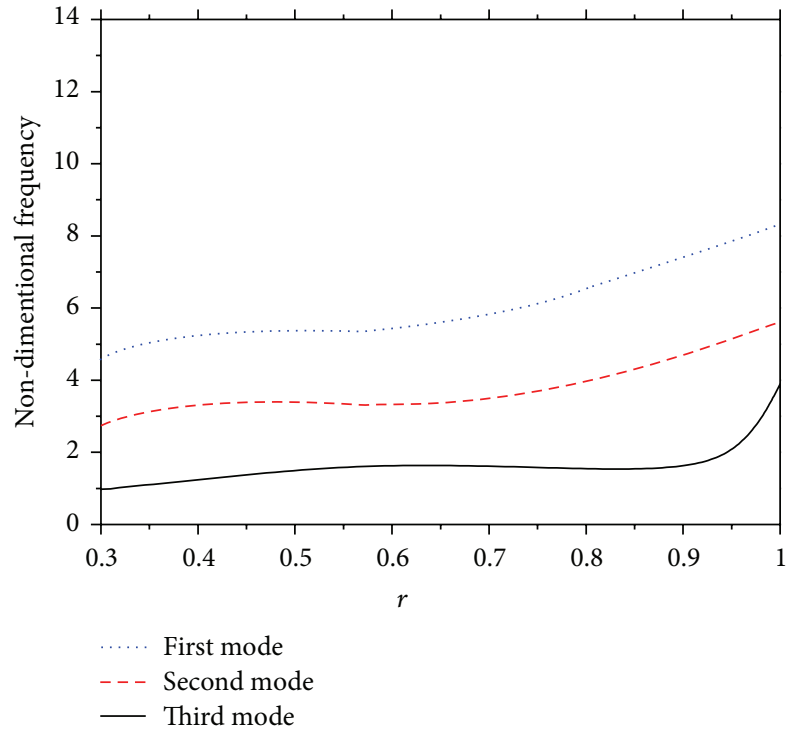

FIGURE 4: Variation of non-dimensional frequency (three modes) versus the radius $r$ of the hollow sphere at rotation $\Omega=2.5$ and non-homogeneous $m=0.65$ (inner fixed surfaces and outer free surfaces) and $n=0$.

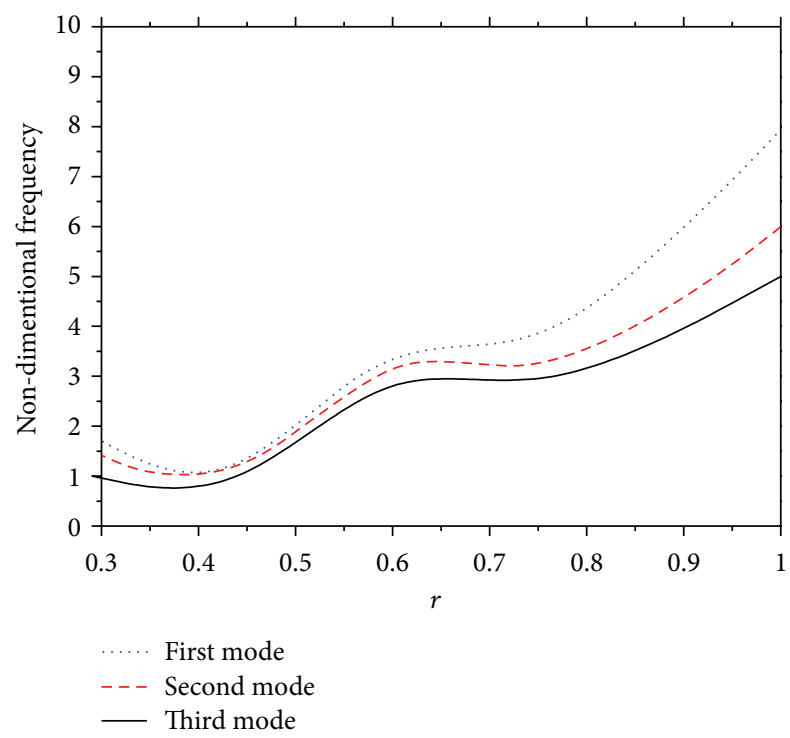

FigURE 5: Variation of non-dimensional frequency (three modes) versus the radius $r$ of the hollow sphere at absent of rotation $\Omega=0.0$ and non-homogeneous $m=0.5$ (inner fixed surfaces and outer free surfaces) and $n=0$.

material. It can be found that the distribution of the nondimensional frequencies is increasing with the increase in the radius $r$, but the non-dimensional frequencies are increasing with the decrease in the rotation. Figure 4 shows the response histories of the non-dimensional frequencies (the first mode, the second mode, and the third mode) with the radius $r$ at value of non-homogeneous $m=0.65$ and the rotation $\Omega=2.5$. It can be found that the distribution of the nondimensional frequencies is increasing with the increase in the

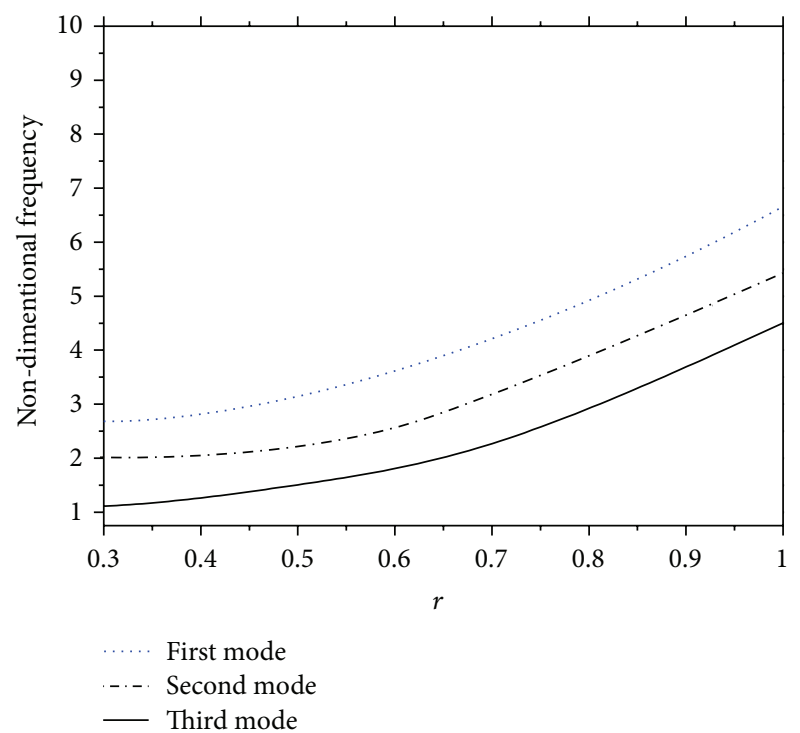

FIGURE 6: Variation of non-dimensional frequency (three modes) versus the radius $r$ of the hollow sphere at rotation $\Omega=2.5$ and nonhomogeneous $m=0.0$ (inner fixed surfaces and outer free surfaces) and $n=0$.

radius $r$, for various boundary conditions, inner fixed surface, and outer free surface. Figure 5 shows the variation of the non-dimensional frequencies (three modes) with the radius $r$ for orthotropic sphere in the absence of rotation $\Omega=0.0$ in the case of non-homogeneous material $m=0.65$. We observed that the frequency is increasing with the increase of the radius $r$ in the case of free traction surfaces, $n=$ 0 . Figure 6 shows the first three modes of non-dimensional frequency for homogeneous $m=0.0$ orthotropic material in presence of the rotation $\Omega=2.5$. We observed that the frequency is increasing with the increase in the radius $r$ in the case of orthotropic homogeneous hollow sphere more than in the case non-homogeneous hollow sphere. It is evident; non-homogenity, rotation, and orthotropic have a significant influence on non-dimensional frequencies.

\section{Conclusion}

The effect of non-homogenity and rotation on surface wave dispersion in elastodynamic problem in orthotropic hollow sphere is studied. The vibration of sphere with the mixed surfaces boundary conditions is evaluated. The natural frequencies (eigenvalues) are calculated and compared with those reported in the absence and presence of non-homogenity and rotation. The effects of non-homogenity and rotation on the natural frequencies are shown graphically.

\section{Acknowledgment}

This work was funded by the Deanship of Scientific Research (DSR), King Abdulaziz University, Jeddah, under grant. The authors, therefore, acknowledge with thanks the DSR technical and financial support. 


\section{References}

[1] S. R. Mahmoud, A. M. Abd-Alla, and N. A. Al-Shehri, "Effect of the rotation on plane vibrations in a transversely isotropic infinite hollow cylinder," International Journal of Modern Physics B, vol. 25, no. 26, pp. 3513-3528, 2011.

[2] S. R. Mahmoud, A. M. Abd-Alla, and M. A. El-Sheikh, "Effect of the rotation on wave motion through cylindrical bore in a micropolar porous medium," International Journal of Modern Physics B, vol. 25, no. 20, pp. 2713-2728, 2011.

[3] S. R. Mahmoud, "Wave propagation in cylindrical poroelastic dry bones," Applied Mathematics \& Information Sciences, vol. 4, no. 2, pp. 209-226, 2010.

[4] M. A. Sumbatyan, V. Tibullo, and V. Zampoli, "Reconstruction of round voids in the elastic half-space: antiplane problem," Mathematical Problems in Engineering, vol. 2006, Article ID 49797, 12 pages, 2006.

[5] V. Seremet, G. Bonnet, and T. Speianu, "New poisson's type integral formula for thermoelastic half-Space," Mathematical Problems in Engineering, vol. 2009, Article ID 284380, 18 pages, 2009.

[6] S. R. Mahmoud, "Effect of rotation and magnetic field through porous medium on peristaltic transport of a Jeffrey fluid in tube," Mathematical Problems in Engineering, vol. 2011, Article ID 971456, 13 pages, 2011.

[7] J. Rungamornrat and P. Tangnovarad, "Analysis of linearly elastic inextensible frames undergoing large displacement and rotation," Mathematical Problems in Engineering, vol. 2011, Article ID 592958, 37 pages, 2011.

[8] A. M. Abd-Alla and S. R. Mahmoud, "Magneto-thermoelastic problem in rotating non-homogeneous orthotropic hollow cylinder under the hyperbolic heat conduction model," Meccanica, vol. 45, no. 4, pp. 451-462, 2010.

[9] A. M. Abd-Alla and S. R. Mahmoud, "On problem of radial vibrations in non-homogeneity isotropic cylinder under influence of initial stress and magnetic field," Journal of Vibration and Control, vol. 19, no. 9, pp. 1283-1293, 2013.

[10] A. M. Abd-Alla, S. R. Mahmoud, S. M. Abo-Dahab, and M. I. Helmy, "Influences of rotation, magnetic field, initial stress, and gravity on Rayleigh waves in a homogeneous orthotropic elastic half-space," Applied Mathematical Sciences, vol. 4, no. 1-4, pp. 91-108, 2010.

[11] A. M. Abd-Alla, S. M. Abo-Dahab, H. A. Hammad, and S. R. Mahmoud, "On generalized magneto-thermoelastic Rayleigh waves in a granular medium under the influence of a gravity field and initial stress," Journal of Vibration and Control, vol. 17, no. 1, pp. 115-128, 2011.

[12] A. M. Abd-Alla, S. R. Mahmoud, and N. A. Al-Shehri, "Effect of the rotation on a non-homogeneous infinite cylinder of orthotropic material," Applied Mathematics and Computation, vol. 217, no. 22, pp. 8914-8922, 2011.

[13] A. M. El-Naggar, A. M. Abd-Alla, and S. R. Mahmoud, "Analytical solution of electro-mechanical wave propagation in long bones," Applied Mathematics and Computation, vol. 119, no. 1, pp. 77-98, 2001.

[14] M. Marin, R. P. Agarwal, and S. R. Mahmoud, "Nonsimple material problems addressed by the Lagrange's identity," Boundary Value Problems, vol. 2013, article 135, 2013.

[15] M. Marin, "Weak solutions in elasticity of dipolar porous materials," Mathematical Problems in Engineering, vol. 2008, Article ID 158908, 8 pages, 2008.
[16] X. Wang, "An elastodynamic solution for an anisotropic hollow sphere," International Journal of Solids and Structures, vol. 31, no. 7, pp. 903-911, 1994.

[17] H. Ding, H. Wang, and W. Chen, "Elastodynamic solution of a non-homogeneous orthotropic hollow cylinder," Acta Mechanica Sinica, vol. 18, no. 6, pp. 621-628, 2002.

[18] H. J. Ding, H. M. Wang, and W. Q. Chen, "A solution of a nonhomogeneous orthotropic cylindrical shell for axisymmetric plane strain dynamic thermoelastic problems," Journal of Sound and Vibration, vol. 263, no. 4, pp. 815-829, 2003.

[19] X. Wang and Y. P. Shen, "Inclusions of arbitrary shape in magnetoelectroelastic composite materials," International Journal of Engineering Science, vol. 41, no. 1, pp. 85-102, 2003.

[20] H. J. Ding, H. M. Wang, and P. F. Hou, “The transient responses of piezoelectric hollow cylinders for axisymmetric plane strain problems," International Journal of Solids and Structures, vol. 40, no. 1, pp. 105-123, 2003.

[21] P. F. Hou and A. Y. T. Leung, "The transient responses of magneto-electro-elastic hollow cylinders," Smart Materials and Structures, vol. 13, no. 4, pp. 762-776, 2004.

[22] G. R. Buchanan and Y. J. Liu, "An analysis of the free vibration of thick-walled isotropic toroidal shells," International Journal of Mechanical Sciences, vol. 47, no. 2, pp. 277-292, 2005.

[23] J. Yu, Q. Ma, and S. Su, "Wave propagation in non-homogeneous magneto-electro-elastic hollow cylinders," Ultrasonics, vol. 48, no. 8, pp. 664-677, 2008.

[24] A. M. Abd-Alla and S. R. Mahmoud, "Analytical solution of wave propagation in a non-homogeneous orthotropic rotating elastic media," Journal of Mechanical Science and Technology, vol. 26, no. 3, pp. 917-926, 2012.

[25] A. M. Abd-Alla, G. A. Yahya, S. R. Mahmoud, and H. S. Alosaimi, "Effect of the rotation, magnetic field and initial stress on peristaltic motion of micropolar fluid," Meccanica, vol. 47, no. 6, pp. 1455-1465, 2012.

[26] S. R. Mahmoud, "Influence of rotation and generalized magneto-thermoelastic on Rayleigh waves in a granular medium under effect of initial stress and gravity field," Meccanica, vol. 47, no. 7, pp. 1561-1579, 2012.

[27] J. N. Sharma, D. K. Sharma, and S. S. Dhaliwal, "Free vibration analysis of a viscothermoelastic solid sphere," International Journal of Applied Mathematics and Mechanics, vol. 8, no. 11, pp. 45-68, 2012.

[28] A. M. Abd-Alla, S. R. Mahmoud, and S. M. Abo-Dahab, "On problem of transient coupled thermoelasticity of an annular fin," Meccanica, vol. 47, no. 5, pp. 1295-1306, 2012.

[29] A. M. Abd-Alla, G. A. Yahya, and S. R. Mahmoud, "Effect of magnetic field and non-homogeneity on the radial vibrations in hollow rotating elastic cylinder," Meccanica, vol. 48, no. 3, pp. 555-566, 2013.

[30] A. M. Abd-Alla, G. A. Yahya, and S. R. Mahmoud, "Radial vibrations in a non-homogeneous orthotropic elastic hollow sphere subjected to rotation," Journal of Computational and Theoretical Nanoscience, vol. 10, no. 2, pp. 455-463, 2013.

[31] A. M. Abd-Alla, S. M. Abo-Dahab, S. R. Mahmoud, and T. A. AlThamalia, "Influence of the rotation and gravity field on stonely waves in a non-homogeneous orthotropic elastic medium," Journal of Computational and Theoretical Nanoscience, vol. 10, no. 2, pp. 297-305, 2013.

[32] A. M. Abd-Alla, S. R. Mahmoud, S. M. Abo-Dahab, and M. I. Helmy, "Propagation of S-wave in a non-homogeneous anisotropic incompressible and initially stressed medium under 
influence of gravity field," Applied Mathematics and Computation, vol. 217, no. 9, pp. 4321-4332, 2011.

[33] A. M. Abd-Alla, S. R. Mahmoud, and S. M. Abo-Dahab, "Wave propagation modeling in cylindrical human long wet bones with cavity," Meccanica, vol. 46, no. 6, pp. 1413-1428, 2011.

[34] T. S. Ozsahin and O. Taskner, "Contact problem for an elastic layer on an elastic half plane loaded by means of three rigid flat punches," Mathematical Problems in Engineering, vol. 2013, Article ID 137427, 14 pages, 2013.

[35] T. H. Daouadji, A. H. Henni, A. Tounsi, and A. B. El Abbes, "A new hyperbolic shear deformation theory for bending analysis of functionally graded plates," Modelling and Simulation in Engineering, vol. 2012, Article ID 159806, 10 pages, 2012.

[36] F. R. Hearmon, An Introduction to Applied Anisotropic Elasticity, Oxford University Press, Oxford, UK, 1961.

[37] R. F. S. Hearmon, "The elastic constants of anisotropic materials," Reviews of Modern Physics, vol. 18, no. 3, pp. 409-440, 1946. 


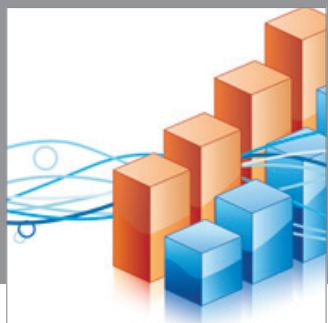

Advances in

Operations Research

mansans

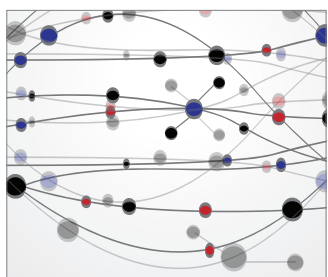

The Scientific World Journal
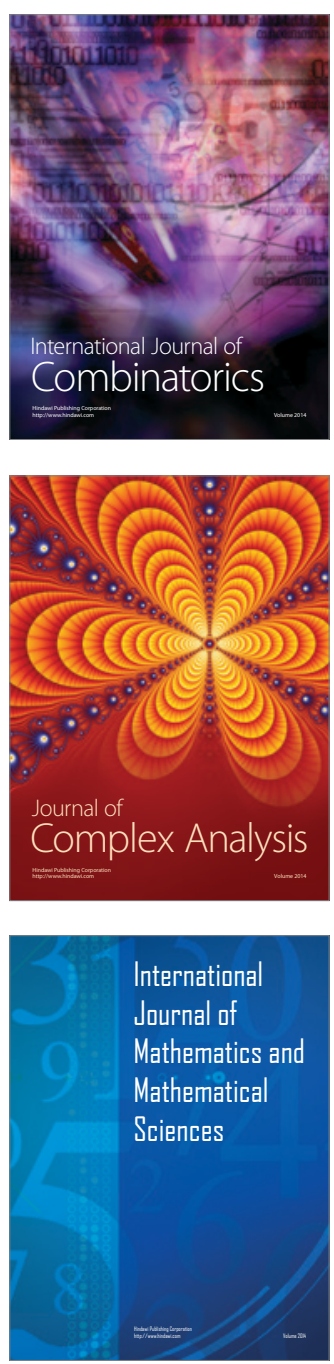
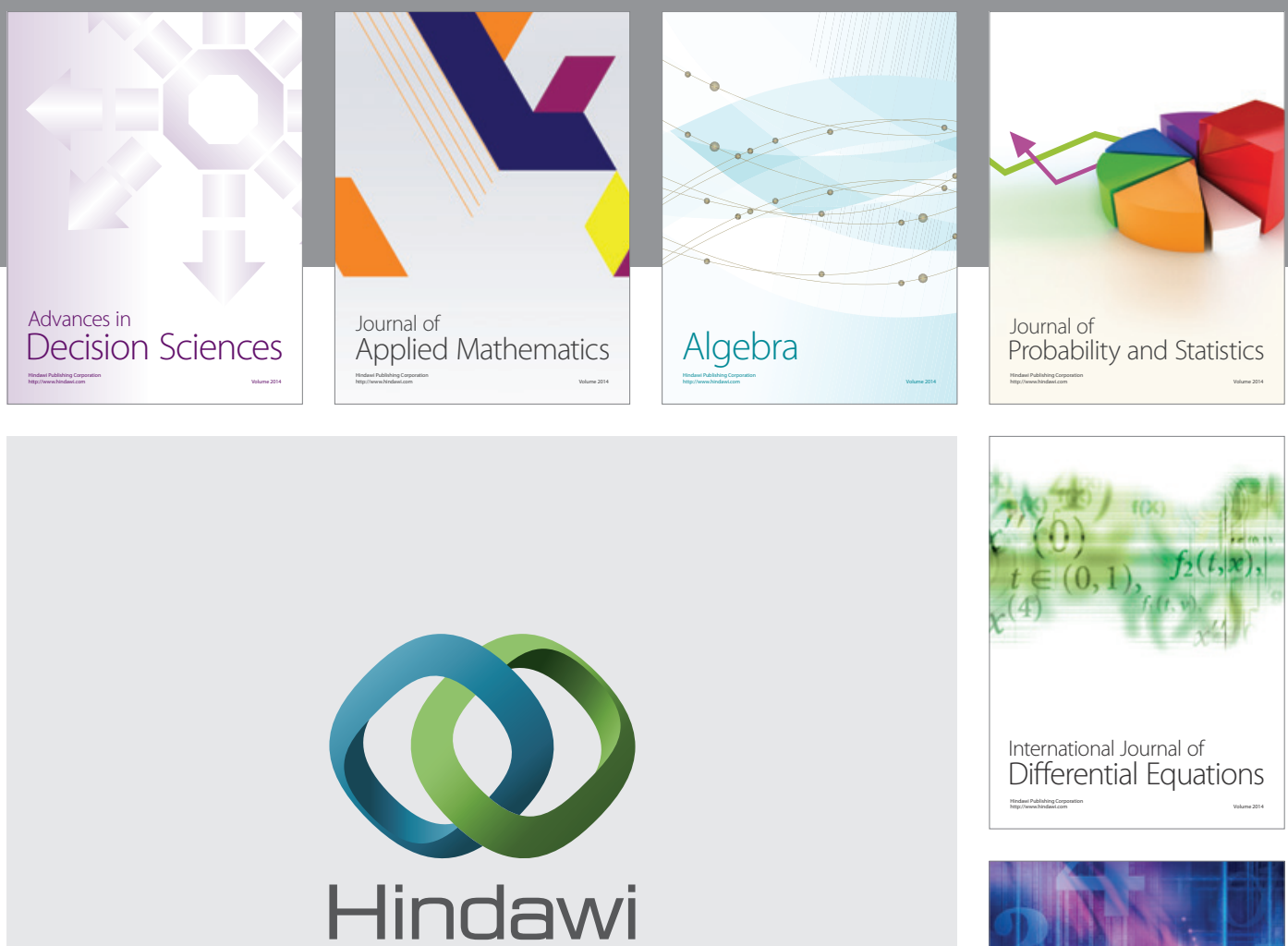

Submit your manuscripts at http://www.hindawi.com
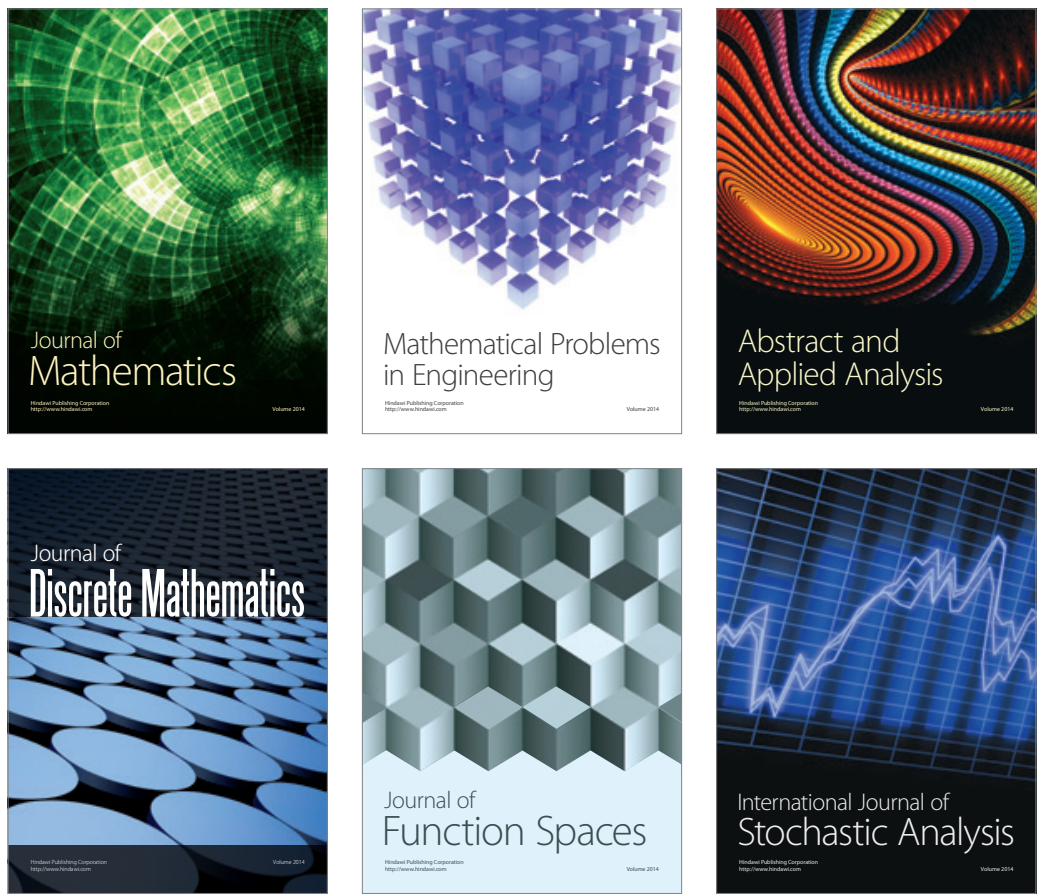

Journal of

Function Spaces

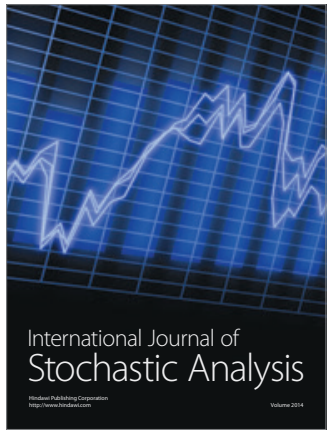

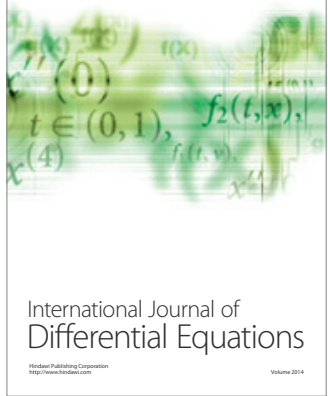
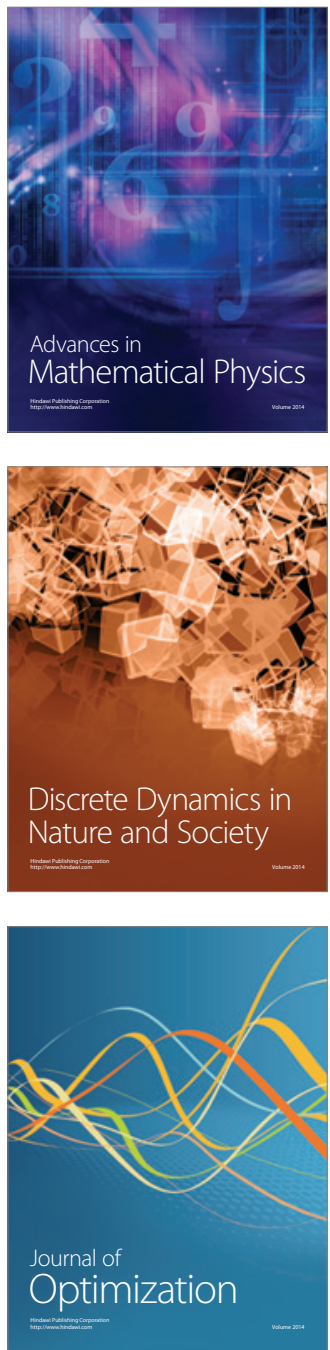